\title{
Morphological Characterization of Antibiotic Combinations
}

Marc A. Coram, ${ }^{\dagger}$ Lisha Wang, ${ }^{\ddagger}$ William J. Godinez, " David T. Barkan, "Zan Armstrong, ${ }^{\dagger}$ D. Michael Ando, ${ }^{*, \dagger, \S}$ and Brian Y. Feng ${ }^{*, \ddagger}, \S$

$\dagger$ Google Research Applied Science, Mountain View, California, 94043, United States $\ddagger$ Infectious Diseases, Novartis Institutes for BioMedical Research, Inc., Emeryville, California, 94608, United States

\Chemical Biology $\&$ Therapeutics, Novartis Institutes for BioMedical Research, Inc., Emeryville, California, 94608, United States

$\S$ These authors have contributed equally. To whom correspondence should be addressed.

E-mail: mando@google.com; brianfeng@calicolabs.com

Table of Contents:

Supplementary methods...(S3)

Data set access...(S5)

Supplementary Table S1...(S6)

Supplementary Figure S1...(S7)

Supplementary Figure S2...(S8)

Supplementary Figure S3...(S9)

Supplementary Figure S4...(S10)

Supplementary Figure S5...(S11)

Supplementary Figure S6...(S12)

Supplementary Figure S7...(S13) 
Supplementary Figure S8...(S14)

Supplementary References...(S15) 


\title{
Supporting Information
}

\author{
Supplementary Methods
}

Processing for single-agent classification validation

Chunking patches into groups of 16

When we process the image of a site in a well, we select a number of image patches spread out over the site. These patches, within each site, were broken, randomly, into groups of 16, discarding any excess. We report classification accuracy at the level of the chunk of patches. The embedding computation computes one vector of numbers per patch. For the analysis reported below, the mean of this vector over a chunk of patches was used to describe the chunk.

\section{Nearest neighbor classification / Train / test split}

To make the train and test datasets balanced, the data was first broken into strata by batch, compound, and concentration (binned to groups of 3 adjacent concentration levels). The number of wells in each stratum were counted; if the count was less than 4 , the stratum was discarded. The led to the exclusion, only one strata: the highest concentration NOVOBIOCIN in the checker01 batch - this condition was retained in the "04" batch. Next, the number of wells to hold out was determined by rounding the count divided by 10 , with a minimum value of 1 . This number of wells per strata was set aside as the hold-out set.

\section{Training a classifier to predict per-patch morphologies}

To refine the well-level letter-code labels into patch-level labels, we followed the following procedure: 1) Tentatively assign the well-level label to every patch in the wells from the well-level supervised subset. 2) Compute embeddings for each patch using the open-source cell-embedder code (https://github.com/google-research/google-research/tree/master/cell_embedder). This 
results in 128 dimensional embeddings (64 per stain). 3) For each label, in turn, collect all embeddings with that label and compute a single best-fitting multivariate Gaussian per label. 4) Construct a formal mixture of these pre-supervised Gaussians, with each component given the weight corresponding to the fraction of patches with that label. 5) Assign new labels to the patches according to the most likely Gaussian component under the mixture model (sklearn.mixture.GaussianMixture's predict method). These resulting labels were summarized to produce the pie-charts describing the letter-codes present in a well.

\section{Clustering morphological checkerboards}

To define a distance matrix between checkerboards, we followed the following procedure: 1) Compute embeddings for each patch, as described above. 2) Take the multivariate Gaussian, described above, that was fitted to the untreated group of patches. 3) Whiten the embeddings by subtracting the mean of this Gaussian and multiplying by the Cholesky decomposition of the precision. 3) For every well, in turn, compute the mean of the whitened patch-level embeddings in that well. 4) In the original checkerboards there are, effectively, 9 doses of compound1 (including the zero dose) combined with 10 doses of compound2 (including the zero dose). Drop the smallest positive dose of compound2, so that there are now 9x9 doses (including the zero doses) in a logical square with a regular progression of fold-MIC. Taking the whitened well-mean embedding vectors for each element of this grid, we obtain a 128x9x9 dimensional matrix. 5) For every pair of checkerboards, in turn, take the corresponding two 128x9x9 dimensional matrices and compute the Euclidean distance between them. Also transpose one of the matrices (with respect to the 9x9 aspect) and compute the Euclidean distance to that transpose. The minimum of these two Euclidean distances was taken to be the distance between the checkerboards. To make the cladogram, the resulting distance

matrix was hierarchically clustered using the Wald criterion and given optimal leaf-ordering. 


\section{Data Set Access}

The image data is available for browsing here:

https://storage.googleapis.com/antibiotic-combination-images/image_browse.html

and are also available for download in Google Cloud. Please use the gsutil app (https://cloud.google.com/storage/docs/gsutil) to download data.

For example, to download the whole data set, type:

gsutil -m cp -r gs://antibiotic-combination-images. Due to the large size of the data set, we recommend first examining the README and manifest files located at gs://antibiotic-combination-images/README.md and gs://antibiotic-combination-images/site_images/manifest.csv, respectively.

The population frequency plots for each checkerboard can be browsed here: https://console.cloud.google.com/storage/browser/antibiotic-combination-images/ checkerboard_pies. The data are organized by plate number and the names of the two agents combined on each checkerboard.

For inquiries about the data, email the corresponding authors: brianfeng@calicolabs. com mando@google.com.

The CI90 checkerboards and their matched morphology checkerboards, summarized at the well level, are available via the journal website. 
Supplementary Table S1: Antibiotics used in this study. ${ }^{*} \mathrm{LpxCi}$ was described as compound $17 \mathrm{x}$ previously. 1

\begin{tabular}{ll}
\hline Antibiotic & Mechanistic Class \\
\hline A22 & cell wall (elongation) \\
AMIKACIN & protein synthesis \\
AMPICILLIN & cell wall (division) \\
AVIBACTAM & cell wall (elongation) \\
AZTREONAM & cell wall (division) \\
BLEOMYCIN & dna damage \\
CEFOXITIN & cell wall (division) \\
CHIR090 & lpxC \\
CHLORAMPHENICOL & protein synthesis \\
CIPROFLOXACIN & dna damage \\
CLINDAMYCIN & protein synthesis \\
DOXYCYCLINE & protein synthesis \\
ERYTHROMYCIN & protein synthesis \\
FUSIDIC ACID & protein synthesis \\
IMIPENEM & cell wall (elongation) \\
LOMEFLOXACIN & dna damage \\
MECILLINAM & cell wall (elongation) \\
MEROPENEM & cell wall (elongation) \\
NALIDIXIC ACID & dna damage (gyrase-ATP) \\
NITROFURANTOIN & dna damage (nitrofuran) \\
NOVOBIOCIN & dna damage (gyrase-ATP) \\
LpXCi* & lpxC \\
PABN & efflux pump \\
PIPERACILLIN & cell wall (division) \\
PMBN & membrane damage \\
RIFAMPICIN & RNA synthesis \\
SPECTINOMYCIN & protein synthesis \\
STREPTOMYCIN & protein synthesis \\
SULFAMETHOXAZOLE & folate biosynthesis \\
SULFAMONOMETHOXINE & folate biosynthesis \\
TETRACYCLINE & protein synthesis \\
TOBRAMYCIN & protein synthesis \\
TRIMETHOPRIM & folatesis \\
\hline
\end{tabular}




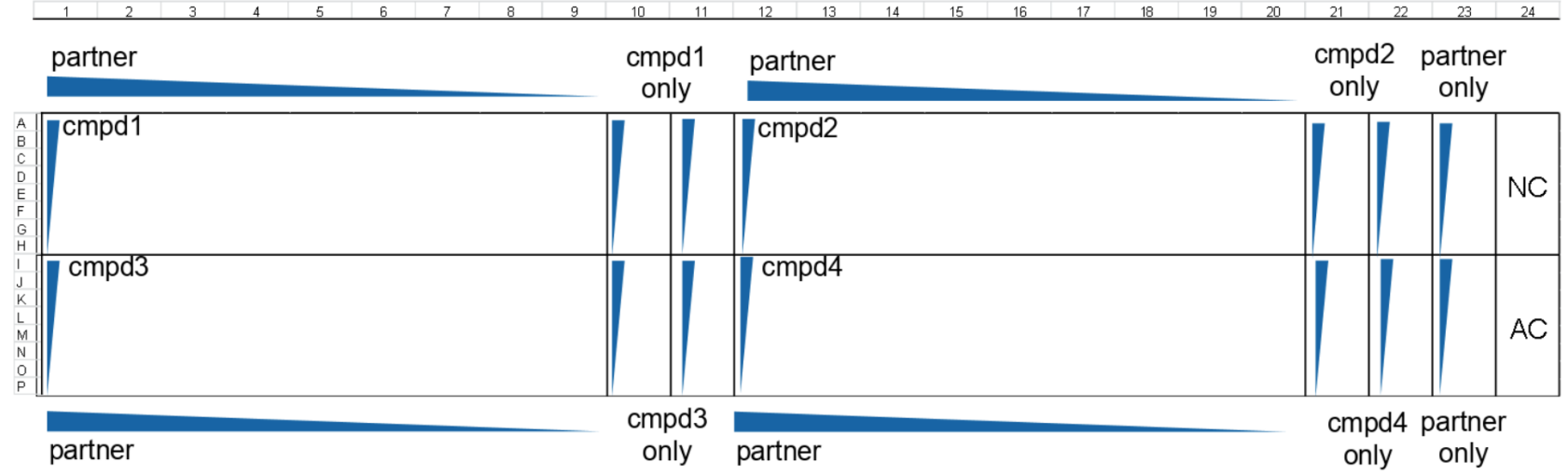

Supplementary Figure S1: Schematic for 384-well format checkerboards. Compounds were tested in 8x9 checkerboard titrations, with each well imaged in at least 6 separate fields. 


\begin{tabular}{|c|c|c|c|c|c|c|c|c|c|c|c|c|c|}
\hline mechanism (target) & cell_wall(division & cell_wall(elongation) & cell_wall(lysis) & dna_synthesis(gyrase-ATP) & ) dna_synthesis(gyrase-FQ) & efflux_pump_inhibitor & r fatty_acid(fabl) & folate_biosynthesis & om_biosynthesis & om_damage & protein_synthesis & ma_synthesis & untreated \\
\hline cell_wall(division) & $30(94 \%)$ & 0 & 0 & \begin{tabular}{|c|}
0 \\
\end{tabular} & \begin{tabular}{|l|} 
\\
\end{tabular} & \begin{tabular}{|l|}
0 \\
\end{tabular} & 0 & 0 & 0 & \begin{tabular}{|c|}
0 \\
\end{tabular} & 0 & 0 & 0 \\
\hline cell_wall(elongation) & 0 & $691(98 \%)$ & 0 & 0 & 4 & 0 & 0 & 0 & 0 & 0 & 0 & 9 & 0 \\
\hline cell_wall(lysis) & 0 & 1 & $14(100 \%)$ & 0 & 0 & 0 & 0 & 0 & 0 & 0 & 0 & 0 & 0 \\
\hline dna_synthesis(gyrase-ATP) & 0 & 0 & 0 & $55(93 \%)$ & 0 & 0 & 0 & 0 & 0 & 1 & 1 & 0 & 0 \\
\hline dna_synthesis(gyrase-FQ) & 0 & 6 & 0 & 1 & $326(99 \%)$ & 0 & 0 & 0 & 0 & 0 & 0 & 3 & 0 \\
\hline efflux_pump_inhibitor & 0 & 0 & 0 & 0 & 0 & $105(100 \%)$ & 0 & 0 & 0 & 0 & 0 & 0 & 0 \\
\hline fatty_acid(fabl) & 0 & 0 & 0 & 0 & 0 & 0 & $10(91 \%)$ & 0 & 0 & 0 & 0 & 0 & 0 \\
\hline folate_biosynthesis & 0 & 0 & 0 & 0 & 0 & 0 & 0 & $126(100 \%)$ & 0 & 0 & 0 & 0 & 0 \\
\hline om_biosynthesis & 0 & 0 & 0 & 0 & 0 & 0 & 0 & 0 & $447(100 \%)$ & 0 & 0 & 0 & 0 \\
\hline om_damage & 0 & 0 & 0 & 1 & 0 & 0 & 1 & 0 & 0 & $260(99 \%)$ & 2 & 0 & 0 \\
\hline protein_synthesis & 2 & 8 & 0 & 2 & 0 & 0 & 0 & 0 & 0 & 1 & $167(97 \%)$ & 0 & 0 \\
\hline ma_synthesis & 0 & 0 & 0 & 0 & 0 & 0 & 0 & 0 & 0 & 0 & 2 & $62(84 \%)$ & 0 \\
\hline untreated & 0 & 0 & 0 & 0 & 0 & 0 & 0 & 0 & 0 & 0 & 0 & 0 & $1138(100 \%)$ \\
\hline
\end{tabular}

Supplementary Figure S2: Confusion matrix expressing the performance of a nearest-neighbor classifier to classify single-agent morphologies based on their embedding vectors. Values along the diagonal are the number and percentage of correct classifications. Off-diagonal values are incorrect classifications. 

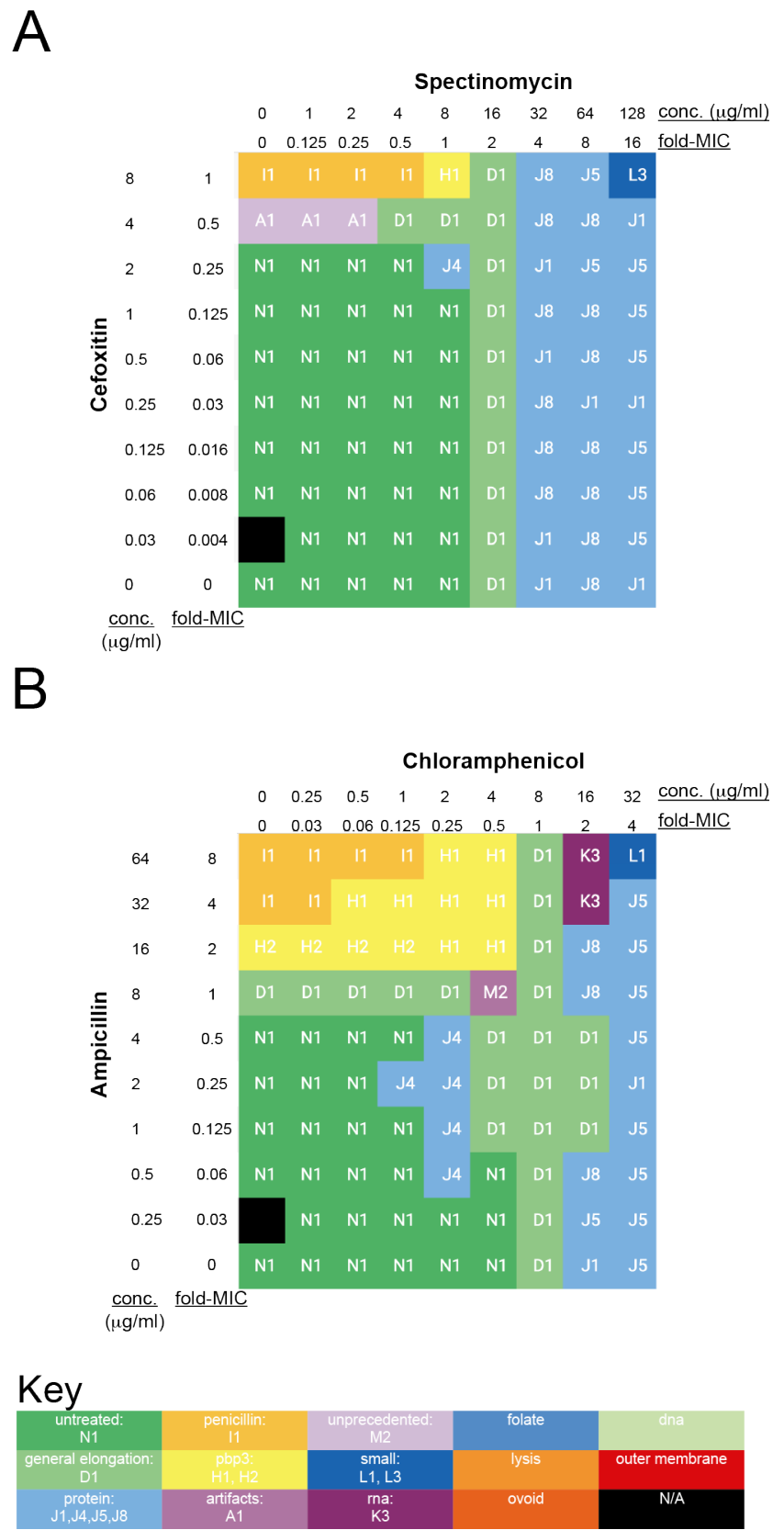

Supplementary Figure S3: Mixtures of Protein synthesis inhibitors and beta-lactam inhibitors preferentially exhibit protein synthesis morphologies over beta-lactam induced lysis phenotypes. 
A (1)

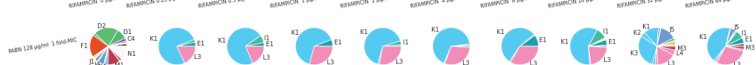

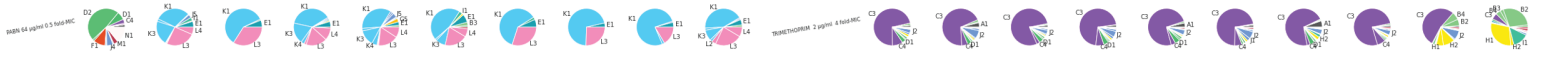
- m.

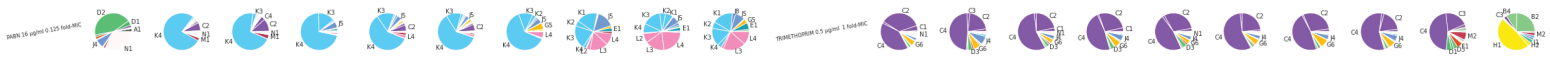

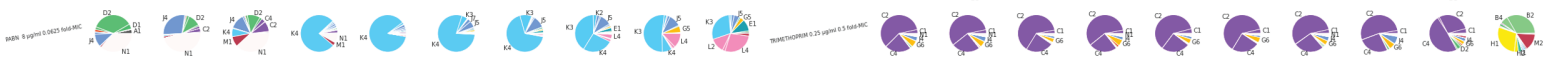
-..... .....

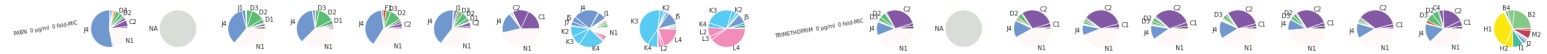

B

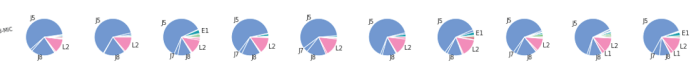

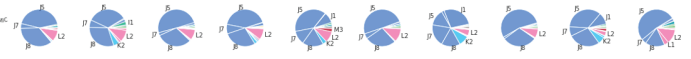
(8)

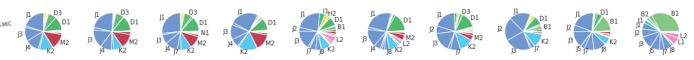
OH.

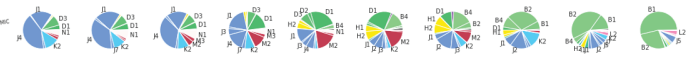
(4) (4) -
D

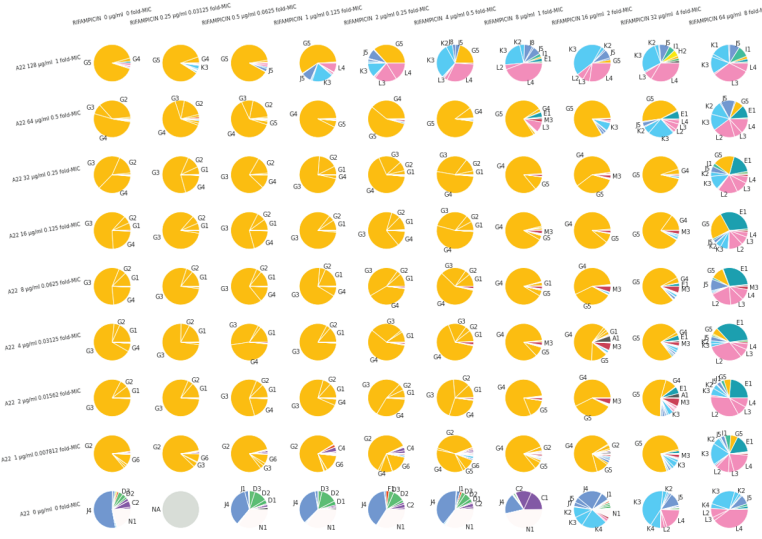

Supplementary Figure S4: Distribution of different morphological phenotypes within each well. A) The distribution of phenotypes for the PABN + rifampin checkerboard from Figure 2A-C. B) The distribution of phenotypes for the tetracycline + ciprofloxacin checkerboard from Figure 2D-F. C) The distribution of the phenotypes for the trimethoprim + cefoxitin checkerboard from Figure 3A-C. D) The distribution of phenotypes from the A22 + rifampin checkerboard from Figure 3D-F. 

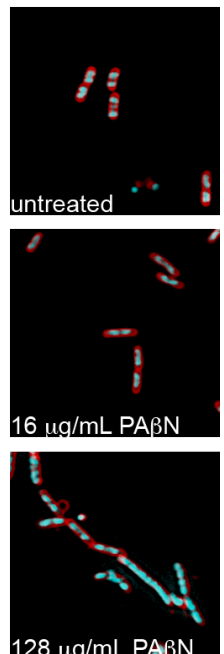

Supplementary Figure S5: At high doses PABN treatment leads to a rare membrane damage morphology. 


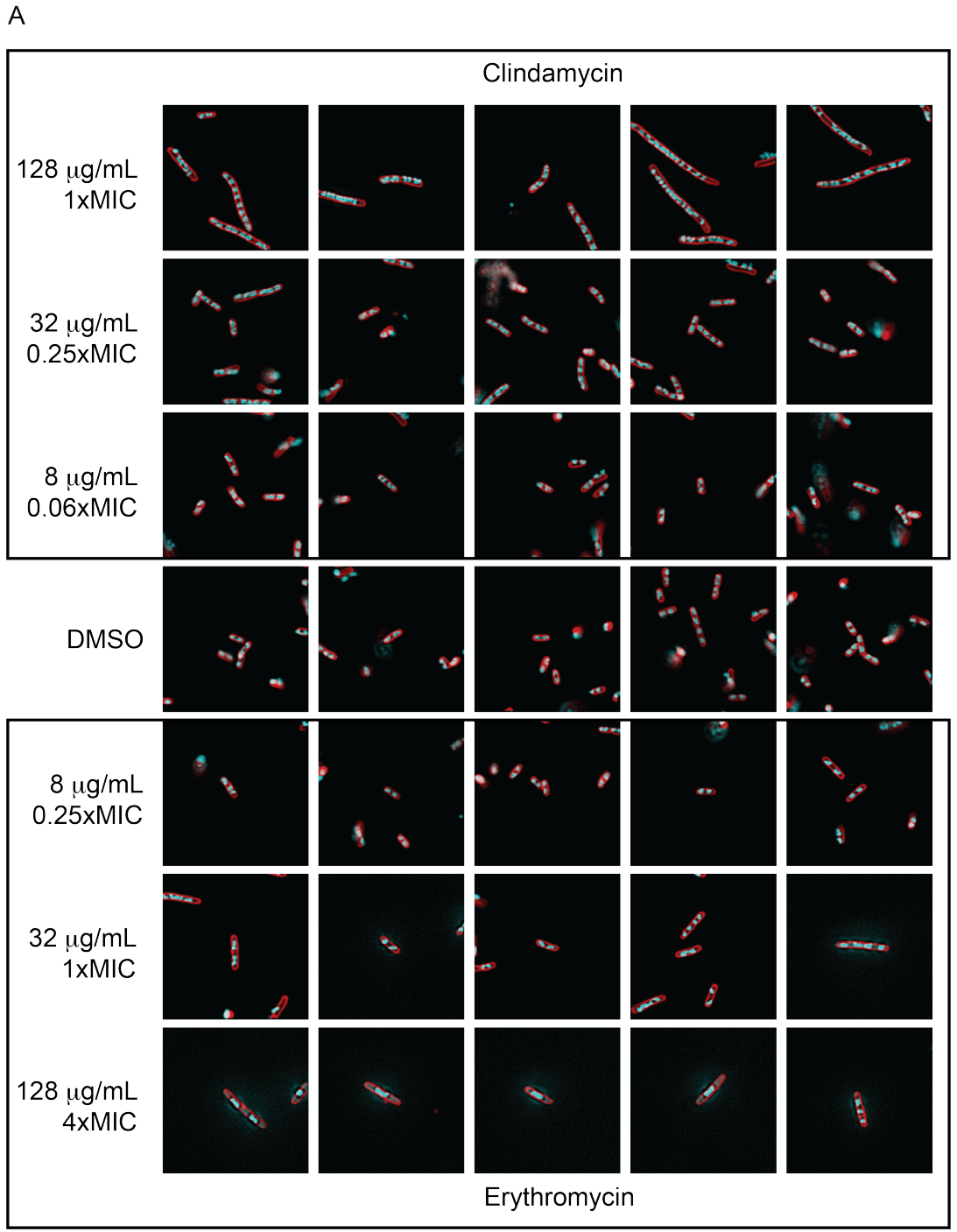

Supplementary Figure S6: Dose-responsive morphological change induced by clindamycin vs. erythromycin. 5 patches from each dose of compound are shown. 


\begin{tabular}{|l|l|l|l|}
\hline Agents & Vendor & Order \# & Lot \# \\
\hline A22 & Sigma & SML0471 & 044M4703V \\
\hline AMIKACIN & USP & 1019508 & JOE226 \\
\hline AMPICILLIN & Sigma & A-9518 & BCBF5293V \\
\hline AVIBACTAM & Novartis & & \\
\hline AZTREONAM & Alfa Aesar & $78110-38-0$ & R21B010 \\
\hline BLEOMYCIN & Calbiochem & 203408 & D00173597 \\
\hline CEFOXITIN & USP & 1098107 & JOE038 \\
\hline CHIRO9O & ChemScene & CS-0973 & 08013 \\
\hline CHLORAMPHENICOL & Calbiochem & 220551 & D00157048 \\
\hline CIPROFLOXACIN & USP & 1134335 & J1L040 \\
\hline CLINDAMYCIN & USP & 1136002 & R011T0 \\
\hline DOXYCYCLINE & Sigma & D-9891 & $093 K 0665$ \\
\hline ERYTHROMYCIN & Sigma & E8755 & $110 K 1168$ \\
\hline FUSIDIC ACID & Sigma & F-0881 & 062K1425 \\
\hline IMIPENEM & USP & 1337809 & I1K226 \\
\hline LOMEFLOXACIN & Sigma & 2906 & $041 \mathrm{M} 1221 \mathrm{~V}$ \\
\hline MECILLINAM & Alfa Aesar & J66774-MC & W30B051 \\
\hline MEROPENEM & USP & 1392454 & JOK434 \\
\hline NALIDIXIC ACID & Sigma & $\mathrm{N}-4382-1 G$ & $106 K 0709$ \\
\hline NITROFURANTOIN & Sigma & $\mathrm{N}-7878$ & \\
\hline NOVOBIOCIN & Sigma & $\mathrm{N}-6160$ & SLBT0910 \\
\hline LPXCi & Novartis & & \\
\hline PABN & Sigma-Aldrich & P4157-25mg & BCBL7201V \\
\hline PIPERACILLIN & USP & 1541500 & JOL275 \\
\hline polymyXIn B nonapeptide & Novartis & & \\
\hline RIFAMPICIN & Sigma & R-3501 & SLBH2676V \\
\hline SPECTINOMYCIN & Sigma & S-9007 & $091 K 1257$ \\
\hline STREPTOMYCIN & USB & 21865 & 122412 \\
\hline SULFAMETHOXAZOLE & USP & 1631001 & J1F148 \\
\hline SULFAMONOMETHOXINE & Sigma-Aldrich & S0508-250mg & $111 \mathrm{M} 4046 V$ \\
\hline TETRACYCLINE & Sigma & T9823 & $081 \mathrm{M} 1598 V$ \\
\hline TOBRAMYCIN & USP & 1667508 & MOM058 \\
\hline TRIMETHOPRIM & Sigma & 46984 & SZB9352XV \\
\hline & & & \\
\hline
\end{tabular}

Supplementary Figure S7: Antibiotics used in this study and their sources. 


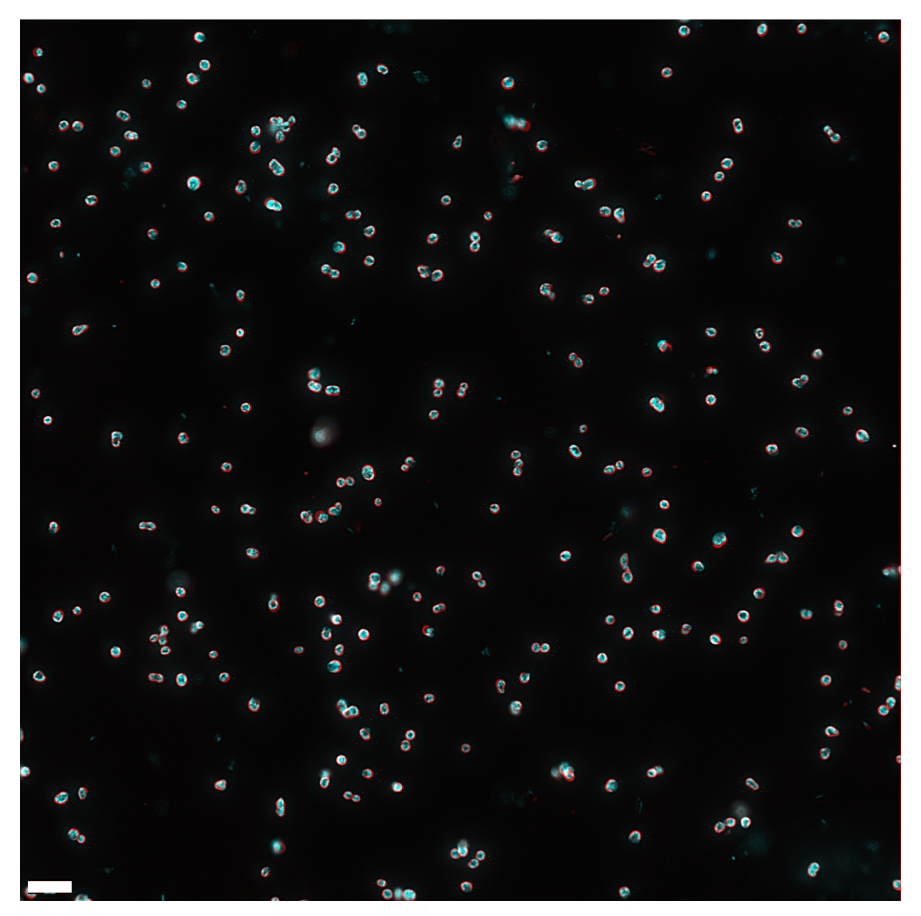

Supplementary Figure S8: An example full field image from the data set. The condition shown corresponds to treatment with $16 \mu \mathrm{g} / \mathrm{mL} \mathrm{A22.} \mathrm{The} \mathrm{red} \mathrm{channel} \mathrm{corresponds} \mathrm{to} \mathrm{FM4-64FX}$ staining of the membrane, the cyan channel corresponds to Hoechst-34580 staining of the nucleoid. The scale bar corresponds to a length of $5 \mathrm{um}$. 


\section{References}

(1) Montgomery, J. I. et al. J. Med. Chem. 2012, 55, 1662-1670. 